\title{
Endometriosis and ovarian cancer risk
}

\begin{abstract}
Epithelial ovarian cancer presents different histological subtypes, mainly serous, mucinous, endometriod, clear cell, mixed and undifferentiated cell. Molecular genetic studies have led to a new paradigm based on a dualistic model of ovarian carcinogenesis. There is a causal association between endometriosis and specific types of ovarian carcinomas, but the magnitude of the risk is low and endometriosis is not considered a premalignant lesion. Among the endometriosis-associated ovarian tumors adenocarcinoma is the most common (Endometrioid and clear cell) (70\%), sarcoma is the second most common malignancy $(12 \%)$ and rare cell types $6 \%$. The gynecologist should pay special attention to identify patients with endometriosis who may be at an increased risk for ovarian cancer.
\end{abstract}

Keywords: epithelial ovarian cancer, gynecologic malignancy, endometriosis, tumor
Volume II Issue 4 - 2020

\author{
Javier de la Torre- Fernandez de Vega, Jose \\ Luis Sánchez-Iglesias, Assumpt Perez- \\ Benavente, Antonio Gil-Moreno \\ Gynaecology Oncology Unit, Hospital Vall d'Hebron, Spain
}

Correspondence: Javier de la Torre Fernández de Vega MD, PhD, Gynaecology Oncology Unit, Hospital Vall d'Hebron, Barcelona, Spain, Email javierdelat@gmail.com

Received: May 31, 2020 | Published: July 222020
Abbreviations: EOC, epithelial ovarian cancer; CCC, clear cell carcinoma; EC, endometrioid carcinoma; EOC, clear cell ca, and endometrioid; MMBT, mullerian or Endocervical-like mucinous borderline tumor; ESS, endometrial stromal sarcomas

\section{Introduction}

Epithelial ovarian cancer (EOC) is the fourth gynecologic malignancy. Around $50 \%$ of female deaths from cancers are due to this tumour. Overall survival at 5 years in epithelial ovarian cancer is $49.7 \%$ according to the 26th volume of the FIGO Annual Report and it varies between $89,6 \%$ in stage IA to $18.6 \%$ for stage IV.

Epithelial ovarian cancer presents different histological subtypes, mainly serous $(70 \%)$, mucinous $(3 \%)$, endometrioid (20-25\%), clear cell $(5-10 \%)$ mixed and undifferentiated cell $(5 \%)$. The histological subtypes of epithelial ovarian cancer look alike the lining of the fimbria of tube, endocervical, digestive epithelium, endometrium, respectively and they are characterized by heterogeneous and display different phenotypes and different molecular characterizations. ${ }^{2,3}$

Molecular genetic studies have led to a dualistic model of origen of EOC. Type I ovarian tumors incorporate high-grade serous carcinomas with TP53 mutations and arise from the epithelium of fimbria. On the other hand, Type II ovarian carcinomas include histological types such as low-grade serous, endometrioid, clear cell, or mucinous carcinomas, and it has been described KRAS or/and BRAF mutations and they may originate from foci of endometrial tissue via retrograde menstruation. ${ }^{4-6}$ There is a meta-analysis that included 7,911 women with ovarian malignancy and concluded that endometriosis is associated with clear cell carcinoma (CCC) (20.2\%), endometrioid carcinoma (EC) (13.9\%), and low-grade serous carcinoma (9.2\%). The risk of malignant transformation of ovarian endometriosis was 2.5 percent.

Sampson, in 1925, put forward a connection between endometriosis and $\mathrm{EOC}^{8}$ and it required a pathological disgnosis of endometriosis or endometrial stroma in close proximity to the tumor.

The risk of ovarian cancer is highest in women with endometriosis and primary infertility. ${ }^{9,10}$ Contrasting to other histologycal types, endometriosis-associated EOC is detected at a younger age and in lower stages. They even have lower grade lesions and a better prognosis. ${ }^{11}$
Although endometriosis cannot be considered as a premalignant disease, it has malignant potential. Moreover, It shares with the cancer similar molecular events, immune disturbances, risk and environmental factors and genetic predisposition. ${ }^{12}$

\section{Molecular Alteration in Endometriosis- associated ovarian tumors}

The molecular events that lead to malignant transformation of endometriosis is heterogeneous and may involve various reported precursor lesions. Inflammation with HNF-1 Beta activation, p53 alteration, PTEN silencing, $\beta$-catenin, KRAS activation mutation, microsatellite instability, ARID1A inactivation mutation, PIK3CAactivating mutations are involved in the occurrence of endometriosisassociated EOC..$^{13,14}$

\section{Types of Endometriosis-associated ovarian tumors}

Between 1966 and 2011, the literature search identified 483 reports describing the association between endometriosis and Ovarian Tumors, $432(89 \%)$ of epithelial malignancy and only 51 (11\%) about mesenchymal malignancy. ${ }^{15} \mathrm{EOC}$ (Clear cell ca, and endometrioid) is the most common (70\%) and different types of sarcoma is the second most common malignancy $(12 \%){ }^{16}$

\section{Endometrioid adenocarcinoma of the ovary}

Although an origen from endometriosis can be demonstrated in the majority of cases it is not required for the diagnosis. These tumors usually express hormone (Estrogen and progesterone) Receptors, suggesting that estrogen could be involved in carcinogenesis. ${ }^{17-19}$ The presence of endometriosis is associated with an increased risk of synchronous neoplasms in the ovary and endometrium and it suggests that they could share the same risk factors for their developmenty. ${ }^{20}$

\section{Clear cell carcinoma of the ovary (CCC)}

Ovarian CCC is composed of glycogen clear cells and typical "hobnail" cells with a low mitotic rate. They are genetically stable and that may contribute to their lack of sensitivity to platinumbased chemotherapy. CCC is divided into two types: Cystic and Adenofibromatous type. Cystic clear cell carcinomas seem to arise from endometrioma, and usually present at early stage, with 
a favorable prognosis. On the other hand, the adenofibromatous type or non-cystic involves the step-wise progression sequence of adenofibromas, atypical proliferative (borderline) tumors, and clear cell carcinoma.

Endometrioid and Clear Cell adenocarcinoma are considered to be "endometriosis-associated ovarian tumors". However, they could comport differently and even arise from different cells of origin. ${ }^{21}$

Additionally, other Müllerian-type borderline tumors could be associated with endometriosis.

\section{Serous borderline tumor}

Typical endosalpingiosis by ectopic growth of ciliated fallopian tubal-type epithelium is frequently found in SBT, but endometriosis was rarely found. Endometrioid adenocarcinoma of the ovary could mimick SBT. ${ }^{22}$

\section{Mullerian or Endocervical-like mucinous borderline tumor (MMBT)}

The mucinous borderline tumors are of intestinal type (85-90\%) or Mullerian type (10-15\%). MMBT is composed of endocervical like mucinous cells and ciliated columnar epithelium of the fimbria, and may also arise from endometriosis. Even the peritoneal implants can arise from foci of endometriosis with transformation. ${ }^{23}$

The join of sarcoma (adenosarcoma and ESS) with endometriosis have also been related.

\section{Adenosarcoma}

Adenosarcoma is an uncommon cancer that shows low malignant potential with a proliferation of a Müllerian- type epithelium with benign, (but occasionally atypical and or malignant) features, mixed with stroma. The tumor is frequently adherent to the surrounding tissue and many cases arise from endometriosis. A major incidence has been seen in women that have received pelvic irradiation and hyperestrogenism, including the use of tamoxifen. ${ }^{24}$

\section{Endometrial stromal sarcoma}

Prymary ovarian endometrial stromal tumors is a very rare monophasic sarcomatous tumour that include stromal nodules, lowgrade endometrial stromal sarcomas (ESS), and undifferentiated endometrial sarcomas. ${ }^{25}$ There have been reported ovarian ESS associated with endometriosis. ${ }^{26}$

\section{Conclusion}

Endometriosis is connected with an increased risk of developing EOC, but it is not considered a premalignant lesion. However, the gynecologist should pay special attention to identify patients with ovarian endometriomas, endometriosis in early age, and associated with infertility which could lead to a higher chance of EOC. Transvaginal ultrasound is useful to identify ovarian endometrioma with mural malignant changes and Magnetic resonance imaging (MRI) may identify malignant transformation of an endometrioma.

Treatment options should be individualized based on the clinical features, patient age, desire for reproduction, and oncological family history. There are no data to indicate that prophylactic extirpation of sites of endometriosis would be useful for reducing the risk of ovarian cancer. However it should be practice in women with complex endometriomas to exclude malignancy associated.
Nowadays, screening women with endometriosis for ovarian cancer is not recommended because of the low incidence and the lack of an effective screening test.

The research should be focused on identifying which women with endometriosis are at risk of developing cancers and to develop preventative measures and curative strategies for this illness.

\section{Acknowledgments}

We thank Angus Powles for all his help and dedication in this paper.

\section{Funding}

None.

\section{Conflicts of interest}

All authors declare that they have no competing interests.

\section{References}

1. Heintz AP, Odicino F, Maisonneuve P, et al. Carcinoma of the ovary. FIGO 26th annual report on the results of treatment in gynecological cancer. Int J Gynaecol Obstet. 2006;95 Suppl 1:S161-S192.

2. Mutch DG, Prat J. FIGO staging for ovarian, fallopian tube and peritoneal cancer. Gynecol Oncol. 2014;133(3):401-404.

3. Sørensen RD, Schnack TH, Karlsen MA, et al. Serous ovarian, fallopian tube and primary peritoneal cancers: a common disease or separate entities - a systematic review. Gynecol Oncol. 2015;136(3):571-581.

4. Lim D, Oliva E. Precursors and pathogenesis of ovarian carcinoma. Pathology. 2013;45(3):229-242.

5. Kurman R, Shih L. The origin and pathogenesis of epithelial ovarian cancer- a proposed unifying theory. Am J Surg Pathol. 2010;34(3):433443.

6. Acién P, Velasco I, Acién M, et al. Epithelial ovarian cancers and endometriosis. Gynecol Obstet Invest. 2015;79(2):126-135.

7. Pearce CL, Templeman C, Rossing MA, et al. Association between endometriosis and risk of histological subtypes of ovarian cancer: a pooled analysis of case-control studies. Lancet Oncol. 2012;13:385-394.

8. Sampson JA. Endometrial carcinoma of the ovary, arising in endometrial tissue in that organ. Arch Surg. 1925;10:1-2.

9. Kumar S, Munkarah A, Arabi H, et al. Prognostic analysis of ovarian cancer associated with endometriosis. Am J Obstet Gynecol. 2011;204:63. e1-67.

10. Wang S, Qiu L, Lang JH, et al. Prognostic analysis of endometrioid epithelial ovarian cancer with or without endometriosis: a 12-year cohort study of Chinese patients. Am J Obstet Gynecol. 2013;209:241.e1-249.

11. Wang S, Qiu L, Lang JH, et al. Clinical analysis of ovarian epithelial carcinoma with coexisting pelvic endometriosis. Am J Obstet Gynecol. 2013;208:413.e1-415.

12. Nezhat F, Apostol R, Mahmoud M, et al. Malignant transformation of endometriosis and its clinical significance. Fertil Steril. 2014;102:342344.

13. Samartzis EP, Noske A, Dedes KJ, et al. ARID1A mutations and PI3K AKT pathway alterations in endometriosis and endometriosis-associated ovarian carcinomas. Int J Mol Sci. 2013;14(9):18824-18849.

14. Okamoto T, Mandai M, Matsumura N, et al. Hepatocyte nuclear factor$1 \beta(\mathrm{HNF}-1 \beta)$ promotes glucose uptake and glycolytic activity in ovarian clear cell carcinoma. Mol Carcinog. 2015;54(1):35-49. 
15. Higashiura $Y$, Kajihara $H$, Shigetomi $H$. Identification of multiple pathways involved in the malignant transformation of endometriosis. Oncology Letters. 2011;4:3-9.

16. Sayasneh A, Tsivos D, Crawford R. Endometriosis and ovarian cancer: a systematic review. ISRN Obstet Gynecol. 2011:14.

17. Gadducci A, Lanfredini N, Tana R. Novel insights on the malignan transformation of endometriosis into ovarian carcinoma. Gynecol Endocrinol. 2014;30(9):612-627.

18. Sieh W, Köbel M, Longacre TA, et al. Hormone-receptor expression and ovarian cancer survival: an Ovarian Tumor Tissue Analysis consortium study. Lancet Oncol. 2013;14(9):853-862.

19. E. Oral, Aydin O, Kumbak BS, et al. Concomitant endometriosis in malignant and borderline ovarian tumours. J Obstet Gynaecol. 2018;8:16.

20. Yamanoi K, Mandai M, Suzuki A, et al. Synchronous primary corpus and ovarian cancer: High incidence of endometriosis and thrombosis. Oncol Lett. 2012;4(3):375-380.
21. Boyraz G, Selcuck I, Yazicioglu A, et al. Ovarian carcinoma associatedwith endometriosis. Eur J Obstet Gynecol Reprod Biol. 2013;170:211-213.

22. Mansor S1, McCluggage WG. Endometrioid adenocarcinoma of the ovary mimicking serous borderline tumor: report of a series of cases. Int J Gynecol Pathol. 2014;33(5):470-476.

23. McCluggage WG. Morphological subtypes of ovarian carcinoma: a review with emphasis on new developments and pathogenesis. Pathology. 2011;43(5):420-432.

24. Gallardo A, Prat J. Mullerian adenosarcoma: a clinicopathologic and immunohistochemical study of 55 cases challenging the existence of adenofibroma. Am J Surg Pathol. 2009;33:278-288.

25. Masand RP1, Euscher ED, Deavers MT, et al. Endometrioid stromal sarcoma: a clinicopathologic study of 63 cases. Am J Surg Pathol. 2013;37(11):1635-1647.

26. Oliva E, Egger JL, Young RH. Primary endometrioid stromal sarcoma of the ovary. Am J Surg Pathol. 2014;38:305-315. 